\title{
AUDAPRET: TOWARDS A CLOUD ARCHITECTURE SUPPORTING MULTI-DEVICE RESEARCH STUDIES
}

\author{
Nadine von Frankenberg, Felix Matschilles and Stephan Jonas \\ Department of Informatics, Technical University of Munich, Germany
}

\begin{abstract}
Recent improvements in the functional range and measurement accuracy of commercially available biosignal measurement devices enable cost-effective human trials with large numbers of participants. Biosignals can be tracked outside the research institution, with only minor restrictions in the daily activities of the study participants, compared to stationary measuring devices. However, there is a lack of data acquisition systems supporting the use of several different devices needed, so that the collected data from measurement devices have to be processed manually. Researchers must also abide by strict legal regulations when handling health-related data of study participants, which is often cumbersome and requires additional time-consuming efforts. To address these challenges, this paper proposes the AUtomatic DAta Processing of REsearch Trials (AUDAPRET) architecture, which enables researchers to conduct trials requiring health-related data of participants in a less time-consuming and legally compliant manner. AUDAPRET is designed in such a way that study participants retain full control over their data and the access rights of anyone requesting access.
\end{abstract}

\section{KEYWORDS}

Human Study Management, Data Collection, eHealth Architectures, Health Information Systems

\section{INTRODUCTION}

With the rise of mobile technologies in the last decade, mobile applications and wearable devices were developed to continuously assess biosignals with increasing accuracy. These advancements have turned health tracking devices into assets for scientific research (Cox et al., 2018), in particular in the health sector. Such research studies often involve a variety of different data sources, especially when the study's focus is on human health: many health-related research studies have successfully been using wearables, the majority of which are activity tracking wristbands (Hartwell et al., 2019). Prominent examples are devices of the brand FitBit, which have been successfully tested in research studies to assess and promote physical activity in cancer and diabetes patients (Coughlin et al., 2020; Zheng et al., 2020). However, studies that require data from multiple different wearables and other sources, such as questionnaires or smart home devices, usually require these data to be collected in a structured and organized manner. Data sources do not necessarily offer uniform interfaces, which makes data collection a challenge. Existing standards, e.g. FHIR or Continua, do not support communication with the participants. Many research institutions follow manual data collection processes, resulting in an additional source of error and workload that increases linearly with the number of participants. Others have used customized systems that were designed specifically for a single study (Aledavood et al., 2017). Developing customized systems for individual studies involves a considerable expenditure of time and budget. While wearable devices appeal to participants due to their minimal restriction on freedom of movement, they also raise privacy concerns (Angeletti et al., 2018). Such devices constantly collect data and may create data profiles about the respective participant's health (Paul and Irvine, 2014). This requires that the data is stored securely and in accordance with the prevailing data protection laws. In reality, this is not always adhered to sufficiently. In Europe, the processing and storage of personal data is regulated by the General Data Protection Regulation (GDPR) (European Commission, 2018): Study participants must be able to give their consent to the use of their data voluntarily, revoke it at any time, and have the right to demand the immediate deletion of their data [GDPR, Art. 7], unless long-term archiving is mandatory, as in clinical trials. The study conductor is obliged to follow such requests immediately [GDPR, 
Article 16]. Health-related data have other specific requirements that must be considered when conducting a research trial, e.g., getting approval of the institutional review board, or follow data processing guidelines.

In this paper, we present the cloud-based AUDAPRET architecture which supports the automation of a data collection process from various data sources for research studies, in a privacy-compliant manner. It is applicable to research studies in any field. The proposed architecture focuses on modularity; additional data sources can be integrated flexibly. To validate the system design and to show its feasibility, a prototype for an exemplary study that integrates data from FitBit, LimeSurvey, and Netatmo has been developed.

\section{RELATED WORK}

In order to conduct research studies, researchers often develop customized systems or workflows that fit to their studies in order to organize and collect multi-source data, which is often time-consuming and cumbersome (Aledavood et al., 2017; Rutkove et al., 2019). Few approaches aim at supporting a multitude of studies and the devices they use to collect and store study-relevant data. Wong et al. conducted a pilot study to test the non-profit software platform Tidepool (https://www.tidepool.org) to automate the data collection in a research study in which diabetic patients were monitored with blood glucose meters. Tidepool focuses on diabetes data and currently only supports data collection wide range of blood glucose meters, making it unsuitable for studies that require data from other wearables, such as EEG headbands or ECG measuring activity trackers. EEG headbands are promising in, but not limited to, studies that aim to record brain activity during epileptic seizures or to investigate cognitive load. Because EEG headbands are unobtrusive, they can be worn outside the clinical environment, which is beneficial because the incidence of seizures is unpredictable and requires continuous monitoring (Byrom et al., 2018).

Niima, the Non-Intrusive Individual Monitoring Architecture platform by Aledavood et al., targets the automation of data collection in research studies to detect and diagnose mental disorders. Niima can be applied to several research areas because of its modular structure. The platform stores data in a raw format and anonymizes these data, before they are accessible to researchers. Study participants do not have access to the data. This restrictive access control leads to a lack of transparency for the participants as to what data are collected in the study as all study participants' data are stored in a central storage (Aledavood et al., 2017).

Angeletti et al. present a software to automate the data collection process in clinical trials. They argue for the need of digital measurements in clinical trials in general, as they noted a decline in people's willingness to participate in studies. This decline indicates that potential study participants often find the time required to participate in studies too long. Their approach specifies that only anonymized or pseudonomized data are stored in their central data storage. Study participants can access the raw sensor data, whereas researchers access the central data storage. However, Angeletti et al.'s solution does not specify the data collection process itself. They argue that the devices used in clinical studies must be certified as clinically useful, but do not propose a solution on how to integrate the devices into their architecture (Angeletti et al., 2018).

The aforementioned approaches focus on different aspects, such as GDPR compliance or the flexibility of integrated data sources. To the best of our knowledge, no existing solution allows researchers to manage data from multiple devices while complying with data protection laws and ensuring transparency for participants.

\section{AUDAPRET}

Our goal was to develop an architecture to assist researchers during data collection and management of research studies, which has to (1) be applicable to different studies with multiple different data sources, (2) comply with data privacy regulations, (3) provide transparency about who can access a participant's data, and (4) allow both researchers and participants to interact. This requirements analysis results from literature research, own experiences, and in consultation with a medical researcher from (REMOVED DUE TO BLIND REVIEW). To address these requirements, AUDAPRET is a decentralized, extensible, cloud-based architecture. It encompasses multiple users of types Researcher and StudyParticipant, each contributing study-relevant data: StudyParticipants provide data from sources such as Wearables or Questionnaires, Researchers contribute data from shared data sources, such as environmental data. AUDAPRET is decomposed into two levels of abstraction: First, the single-user level defines the interaction between each 
user and their dedicated instance of the SingleUserSubsystem, which collects the user's study relevant data and allows researchers to access this data. In addition to their SingleUserSubsystem instance, Researchers use the StudyAdministrator to manage a study and to request data from StudyParticipants. Second, the multi-user level covers the interaction between multiple SingleUserSubsystem instances and the StudyAdministrator.

The SingleUserSubsystem consists of three subsystems (see Figure 1): The StudyDataManager stores and administrates study-relevant data of a user. The user interacts with the StudyDataManagerClient to view, delete or anonymize StudyData of the respective StudyDataManager. An Adapter binds a DataSource to the StudyDataManager; it independently collects data from its DataSources, processes it, and sends it to the StudyDataManager. The StudyDataManager passively receives and stores data from all its Adapters and returns all data on request. Adapters are bound to the study's domain, whereas the StudyDataManager and the StudyAdministrator operate independently and can therefore be reused in studies of any domain.

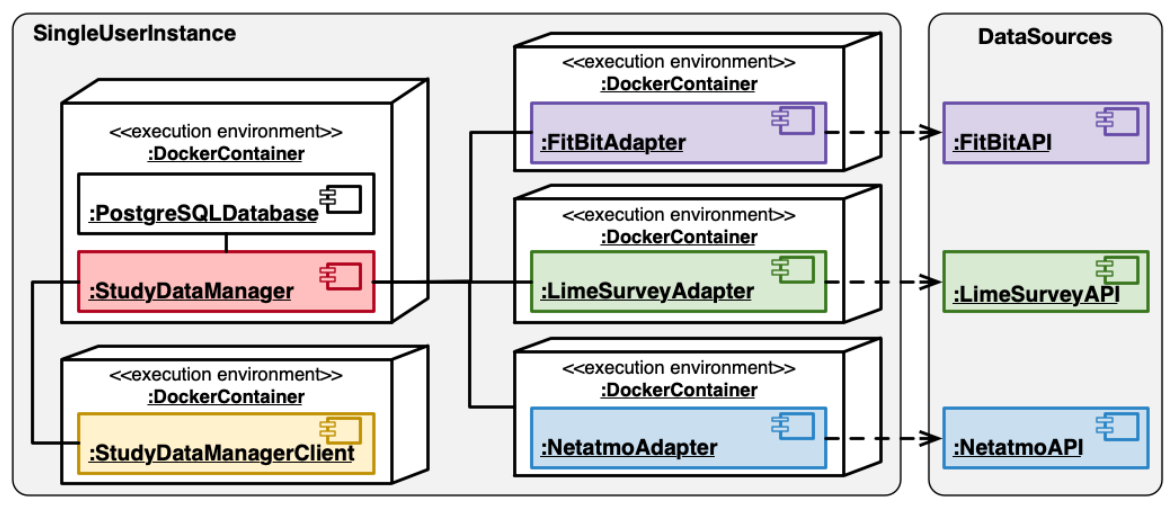

Figure 1. Subsystem decomposition and prototype adapters of the SingleUserInstance

We have developed a prototype of AUDAPRET, where all individual subsystems are implemented in TypeScript. The prototype integrates Adapters for activity trackers (FitBit), questionnaires and surveys (LimeSurvey), and indoor weather stations (Netatmo). It addresses a concrete instance of the SingleUserSubsystem as SingleUserInstance, consisting of a StudyDataManager, StudyDataManagerClient, and the domain-dependent Adapters FitBitAdapter, LimeSurveyAdapter, and NetatmoAdapter. Each Adapter is deployed in a separate docker container so that the Adapters will continue to collect data even in the event of an API malfunction. The containers of the StudyDataManager, StudyDataManagerClient, and Adapters can be composed to an isolated network, in which each Adapter's container is linked to the StudyDataManager. Each subsystem server of a SingleUserInstance can be deployed in a web cloud in its own container, and composing it into a container network, the SingleUserInstance can be duplicated to any number with container orchestration tools, such as Kubernetes.

At the multi-user level, AUDAPRET consists of multiple independently deployed SingleUserInstances, one per user. Additionally, Researchers use the StudyAdministrator which allows them to administrate a study and to request data from all StudyParticipants of this study. The StudyAdministrator communicates with the SingleUserSubsystem instances of the respective StudyParticipants (see Figure ).

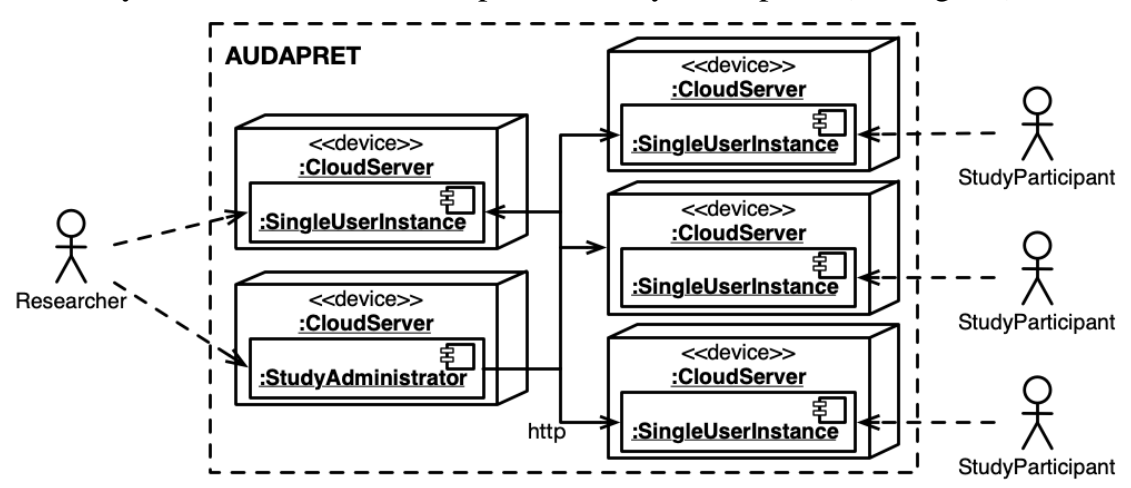

Figure 2. Subsystem decomposition of an exemplary study on the multi-user level as an UML diagram 
If personal data is collected and stored as part of a research trial, StudyParticipants have the right to access and delete their own data at any time (European Commission, 2018). Furthermore, Researchers who store data are liable for violations against the GDPR. By using localized or personalized instances, data ownership is delegated to the StudyParticipant. Thus, each StudyParticipant has their own database which stores their respective study data, and which is initially only accessible to the respective StudyParticipant. If a Researcher wishes to access a StudyParticipant's data for evaluation purposes, they can request access to read the StudyParticipant's data. This enables the StudyParticipant to view, delete, and anonymize their data at any time and therefore does not require the Researchers to offer this service to the StudyParticipants.

\section{CONCLUSION}

AUDAPRET supports the conduct of human studies: It automatically collects data from multiple sources, e.g., questionnaires, wearables, and environmental sensors. It can be easily extended to collect data from additional sources and can be used in studies from different fields that require data from different sources. The AUDAPRET system is designed so that data collection, processing, and storage is GDPR-compliant, while Study-Participants have full control over their personal StudyData. StudyParticipants can share their StudyData voluntarily with Researchers and are able to view, delete or anonymize their data at any time. As proof of concept, we have implemented a prototype of the system which supports AUDAPRET's main functionality: collecting data and sharing it with the Researcher on the participant side and requesting this data on the researcher side. The prototype collects data from FitBit, LimeSurvey, and Netatmo.

For future work, we intend to validate AUDAPRET in a multi-participant experiment, where each participant contributes data from multiple sources. The current design of a participant-centered data storage allows StudyParticipants to retain their StudyDataManager and StudyData after the end of the study. Since they own their personal StudyData, they could share them with other Researchers for other research projects. We envision a platform that enables StudyParticipants to share their StudyData with other Researchers using the same access grant logic as designed for a single study. Other existing mHealth applications, whose core functionality is the collection of health data, could also be integrated into AUDAPRET as data sources.

\section{REFERENCES}

Aledavood, T., Hoyos, A.M.T., Alakörkkö, T., Kaski, K., Saramäki, J., Isometsä, E., Darst, R.K., 2017. Data collection for mental health studies through digital platforms: requirements and design of a prototype. JMIR Res. Protoc. 6, e110.

Angeletti, F., Chatzigiannakis, I., Vitaletti, A., 2018. Towards an architecture to guarantee both data privacy and utility in the first phases of digital clinical trials. Sensors 18, 4175.

Byrom, B., McCarthy, M., Schueler, P., Muehlhausen, W., 2018. Brain monitoring devices in neuroscience clinical research: the potential of remote monitoring using sensors, wearables, and mobile devices. Clin. Pharmacol. Ther. 104, 59-71.

Coughlin, S.S., Caplan, L.S., Stone, R., 2020. Use of consumer wearable devices to promote physical activity among breast, prostate, and colorectal cancer survivors: a review of health intervention studies. J. Cancer Surviv. 1-7.

Cox, S.M., Lane, A., Volchenboum, S.L., 2018. Use of wearable, mobile, and sensor technology in cancer clinical trials. JCO Clin. Cancer Inform. 2, 1-11.

European Commission, 2018. 2018 reform of EU data protection rules.

Hartwell, L., Ross, H.M., La Belle, J.T., 2019. Project honeybee: Clinical applications for wearable biosensors. Biomed. Microdevices 21, 37.

Paul, G., Irvine, J., 2014. Privacy implications of wearable health devices. In: Proceedings of the 7th International Conference on Security of Information and Networks. pp. 117-121.

Rutkove, S.B., Qi, K., Shelton, K., Liss, J., Berisha, V., Shefner, J.M., 2019. ALS longitudinal studies with frequent data collection at home: study design and baseline data. Amyotroph. Lateral Scler. Front. Degener. 20, 61-67.

Zheng, Y., Weinger, K., Greenberg, J., Burke, L.E., Sereika, S.M., Patience, N., Gregas, M.C., Li, Z., Qi, C., Yamasaki, J., 2020. Actual Use of Multiple Health Monitors Among Older Adults With Diabetes: Pilot Study. JMIR Aging 3, e15995. 